\title{
$5 \%$ lidocaine medicated plaster double effect in a case of orofacial localized neuropathic pain
}

This article was published in the following Dove Press journal: Journal of Pain Research

24 November 2014

Number of times this article has been viewed

Roberto Casale ${ }^{1,2}$

Yuriy Romanenko 2,3

Massimo Allegri ${ }^{4-6}$

'Department of Clinical Neurophysiology and Pain Rehabilitation Unit, Foundation "Salvatore Maugeri", Research and Care Institute, IRCCS, Pavia, Italy; ${ }^{2}$ EFIC Montescano Pain School, Montescano, Italy; ${ }^{3}$ Department of Neurology, Lugansk City Hospital 4, Lugansk, Ukraine; ${ }^{4}$ Department of Surgical Science, University of Parma, Parma, Italy; ${ }^{5}$ Pain Therapy Service, Azienda Ospedaliera Universitaria Parma, University of Parma, Parma, Italy; ${ }^{6}$ Study In Multidisciplinary Pain Research Group, Pavia, Italy
Correspondence: Roberto Casale Department of Clinical Neurophysiology and Pain Rehabilitation Unit, Foundation "Salvatore Maugeri", Research and Care Institute of Montescano, Via per Montescano, 27040 Montescano, Pavia, Italy

Tel +390385247249

Fax +390385247336

Email roberto.casale@fsm.it
Abstract: Localized neuropathic pain (LNP) is a type of neuropathic pain that is characterized by "consistent and limited area(s) of maximum pain associated with negative or positive sensory signs and/or spontaneous symptoms characteristic of neuropathic pain". This definition encompasses a huge number of neuropathic orofacial pain syndromes. We present a case report of a patient who was affected with sleep apnea syndrome treated with nocturnal oxygen mask delivery, in whom orofacial LNP hampered the wearing of a mask due to unbearable burning and throbbing pain. The application of 5\% lidocaine medicated plaster during the night led to an impressive reduction of both the pain level and the size of the painful area due to the plaster's pharmacological mechanisms, which were associated with a secondary benefit due to its mechanical protective action. This case report shows how these two factors could be of clinical value and have to be considered more systematically in the treatment of LNP in reducing pain and the size of the painful area.

Keywords: trigeminal pain, localized neuropathic pain, topical treatment, 5\% lidocaine medicated plaster

\section{Introduction}

Within the broad definition of neuropathic pain, ${ }^{1}$ the refinement of clinical diagnostic procedures has led to the introduction of localized neuropathic pain (LNP), characterized by "consistent and limited area(s) of maximum pain associated with negative or positive sensory signs and/or spontaneous symptoms characteristic of neuropathic pain". ${ }^{2}$ This definition also encompasses a huge number of neuropathic orofacial pain syndromes (NOP). Guidelines for LNP suggest local treatments as a first-line treatment. ${ }^{3,4}$ Lidocaine in the periphery exerts its action by binding to the sodium channels located on free nerve endings that transmit pain, blocking the abnormal sodium channels present in higher numbers on hyperactive or damaged nociceptors. ${ }^{5,6}$

$5 \%$ lidocaine medicated plaster has been recently reviewed and suggested for the treatment of neuropathic pain of peripheral origin, other than postherpetic neuralgia and painful diabetic neuropathy. ${ }^{7}$ Currently, no specific indications or case reports have been published for 5\% lidocaine treatment for orofacial LNP (L-NOP). Even though the most common mechanism of L-NOP is deafferentation (sensory loss), in some cases, the injuries produce positive symptoms with peripheral neuropathic pain. Paroxysmal neuropathies characterized by shooting or sharp pain are typical for trigeminal neuralgia, while continuous pain, sometimes of a burning quality, is a characteristic of post-traumatic neuropathy ${ }^{8-10}$ Furthermore, thermal and mechanical allodynia are frequently associated with peripheral L-NOP. ${ }^{8-10}$ 
Although these symptoms are thought to be pivotal targets for medical treatments, they are generally weighted only in terms of the intensity of pain. Information on the reduction of the size of the painful area are poor and scattered in the literature, and the possible mechanical protective efficacy of the plaster itself has been poorly investigated.

This is a case report of an L-NOP that occurred following dental surgery in a patient affected by sleep apnea syndrome treated with a nocturnal oxygen mask, in whom both the active compound and the mechanical protection of a 5\% lidocaine medicated plaster played an important role in pain control and in the improvement of quality of life through the plaster's properties in reducing pain intensity and the size of the affected area.

\section{Case report}

GC, a 58-year-old man affected by sleep apnea syndrome treated with continuous positive airway pressure nocturnal oxygen therapy (cPAP), underwent dental surgery in the right mandibular area in 2011 for dental implantation. Several troncular anesthetic injections of the inferior alveolar (dental) nerve at the mandibular angle were performed. During one of these injections, CG reported the sudden onset of acute shooting pain felt in the area of the homolateral mental nerve at the chin and the third distal part of the mandibular border. In the same area, once the local anesthesia wore off, he started to complain of burning pain associated with severe static mechanical allodynia and hyperalgesia. The pain was so severe that it would not to allow him to wear his oxygen cPAP mask.

In the following months, several pharmacological treatments, including anti-inflammatory, serotonergic antidepressants alone (duloxetine up to $120 \mathrm{mg}$ ) and in combination with antiepileptic drugs, gabapentinoids (pregabalin up to $600 \mathrm{mg}$ ), and weak and strong opiates, were administered without any improvement. Uncontrolled pain associated with diurnal drowsiness, tiredness, and a lack of concentration induced by a lack of restorative sleep and the untreated hypoxia, greatly affected his work, inducing reactive depression and resulting in a dramatic decrease in his quality of life. For these reasons, GC spontaneously interrupted all pharmacological pain treatments, except for the antidepression treatments (duloxetine [90 mg/day] plus alprazolam [0.75 mg/day]).

At the time of our first consultation, 2 years after the onset of pain, GC reported continuous burning pain associated with mechanic allodynia, overlapping the causalgic area in the cutaneous territory of the right mental nerve at the chin, as well as the third distal part of the mandibular border. He reported that after a while, the pain spread to the right angle of the mouth and upper lip (Figure 1A). Pain intensity was evaluated as "more than 10" on the 0-10 cm Visual Analog Scale (VAS). A quantitative sensory test evaluation of the area, compared with the contralateral side, showed thermal allodynia in the area of the reported symptoms. No other neurophysiological assessments were done because of the strong painful reaction to any stimulation. The patient's quality of life was markedly affected with a EuroQol value of $0.64 .{ }^{11}$

The presence of a clinical history plausible for a mandibular alveolar nerve lesion, as well as the clinical findings, were indicative of L-NOP. Hence, we started with 5\% lidocaine medicated plaster treatment (12 hours a day).

The patient was informed that at the time of treatment, no specific indications had been published for $5 \%$ lidocaine medicated plaster for L-NOP. For this reason, written informed consent was obtained from the patient both for the treatment, and separately for the publication of this case report and any accompanying images.

For practical reasons, we first decided to apply the plaster to the area(s) of maximal pain during the day. After 14 days of treatment, spontaneous pain intensity was measured as a score of 6.7 on the VAS, accompanied by a marked reduction in the painful area (Figure 1B). Upon neurological evaluation with a Von Frey hair (4.56 g corresponding to a target force of 4 grams [Touch-Test ${ }^{\circledR}$ Sensory Evaluation; North Coast Medical Inc., Gilroy, CA, USA]), we also observed a reduction in the mechanical allodynic area. Moreover, the appearance of an area of numbness was assessed with Von Frey hair. ${ }^{12}$ However,

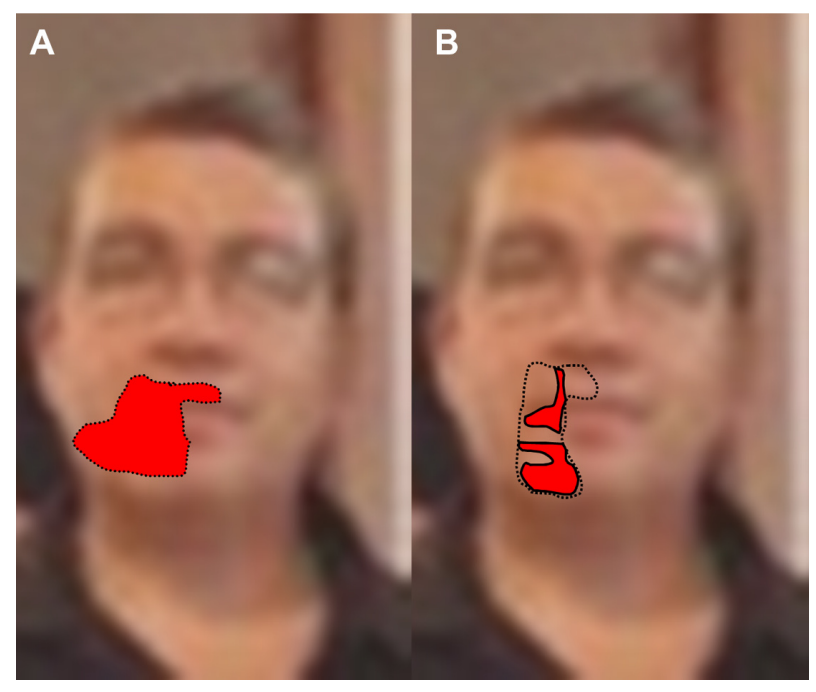

Figure I Spontaneous burning pain and numbness associated with allodynia. Notes: (A) June 17, 2013. Red area: spontaneous burning pain associated with allodynia to mechanical stimuli. (B) July I, 2013. Reduction of the size of the red area and of symptom intensity. At control, the pain was not only reduced in terms of intensity and the size of the area, but sensory changes were also observed with a patchy distribution of numbness (not colored), and allodynic areas (red areas) were still present. 
cPAP application was still problematic, as the patient did not tolerate the oxygen mask longer than 30 minutes to 1 hour due to the presence of unbearable burning and throbbing pain in the remaining painful area. Hence, we decided to apply the plaster at night during cPAP therapy, so as to also ensure that the patient received mechanic protection from mask compression on the allodynic area. The results were immediately positive, as it was possible for the patient to complete the polysomnographic studies and to correct the nocturnal hypoxia.

Currently, GC is still using 5\% lidocaine medicated plaster at night with good pain control (the VAS was 4.5) when he is under cPAP, with a pressure of $16 \mathrm{~cm} \mathrm{H}_{2} \mathrm{O}$. His antidepressant therapy is in progressive reduction (duloxetine to $30 \mathrm{mg}$ /day and alprazolam to $0.25 \mathrm{mg} /$ day). His quality of life also showed a dramatic improvement to 0.87 on the EurQol scale. No systemic or local side effects have been reported thus far.

\section{Discussion}

LNP can occur in the orofacial region, not only as a painful consequence of herpetic or trigeminal neuralgias, but also as a result of nerve injuries after dental surgery. Unfortunately, the epidemiological data for L-NOP are quite scant and unknown, even though some studies estimated that L-NOP affects approximately $3 \%-6 \%$ of the population. ${ }^{8,10}$ This case report pinpoints the usefulness of $5 \%$ lidocaine medicated plaster treatment when all the diagnostic steps for a correct diagnosis of LNP are fulfilled: the presence of a nerve lesion; and positive symptoms referred on the skin in a circumscribed area with a predominance of a spontaneous burning sensation. ${ }^{13}$

This case also indicates the possible reduction of the size of the painful area induced by targeted treatment with $5 \%$ lidocaine medicated plaster, and it shows the three clinical aspects of pain and pain relief distribution in L-NOP: pain felt in areas not related to the mandibular nerve; the patchy reduction of the painful area; and the clinical appearance of negative sensory symptoms. ${ }^{14,15}$ The area of reported pain spread outside the territory of the lesioned nerve (ie, the right mandibular nerve, third trigeminal branch - V3) toward contiguous territory innervated by the second trigeminal branch (V2). Indeed, in the trigeminal system, the substantial convergence of afferent somatosensory inputs from different anatomical structures at the level of the spinal trigeminal subnucleus caudalis has been described. ${ }^{16}$ This can account for the distribution of the painful area outside the V3 trigeminal branch. ${ }^{16}$ Consistent with nonpredictable and nonuniform mandibular nerve injury, the patchy nature of the reported reduction in the painful area can also be attributed to a nonhomogeneous distribution of cutaneous pain generators within the painful area. ${ }^{13}$ The reduction of pain intensity and the size of the painful area induced by the application of 5\% lidocaine medicated plaster had unmasked negative sensory disturbances that were not otherwise clinically evident initially. In this case report, the negative sensory disturbances (typical of a peripheral nerve lesion) confirmed the neuropathic origin of the pain and the presence of a LNP. These negative sensory disturbances cannot be attributed to hypothetical anesthetic action. Prior studies in healthy volunteers did not show any loss of mechanical sensation at the application site. ${ }^{17}$ In the literature on LNP, the presence of negative symptoms unveiled by the local application of $5 \%$ lidocaine plaster has not been sufficiently explored.

Lidocaine led to a pain relief score of 3.3 on the VAS; moreover, the protective mechanical action of the plaster itself appears to provide special therapeutic and protective action toward continuous mechanical stimulation resulting from the cPAP mask, resulting in an additional 2.2 points of improvement on the VAS. ${ }^{14}$ The mechanical protection of the plaster itself that is associated with the pharmacological action of the lidocaine can also exert a "secondary analgesic effect", reducing the mechanical stimulation of the already sensitized peripheral receptors, thus leading to a reduction of spinal cord excitability (windup and central sensitization). ${ }^{18}$

Improvement in the patients' health-related quality of life, previously shown in a study on postherpetic neuralgia over a 12 -week $5 \%$ lidocaine medicated plaster treatment, is herein confirmed. ${ }^{11}$

\section{Conclusion}

To our knowledge, this is the first report of the successful treatment of L-NOP with 5\% lidocaine medicated plaster. This report shows not only the treatment's efficacy in controlling pain intensity, but it also shows other therapeutic targets - namely, the reduction of the painful area - which has only occasionally been reported with the use of systemic drugs, such as gabapentin. ${ }^{19}$ These targets include:

1. reduction of the painful area;

2. mechanical protective action of the allodynic area;

3. possible positive effects on central sensitization (reduction); ${ }^{20}$ and

4. the reduction of side effects, as well as high adhesion and compliance to the treatment, thus improving the quality of life.

In the near future, these aspects of targeted topical treatments for both LNP and L-NOP should be systematically studied in clinical trials that are expressly designed to investigate these endpoints. 


\section{Author contributions}

Dr Casale has made substantial contributions to the conception and design, acquisition of data, and analysis and interpretation of data; he has been involved in drafting the manuscript, revising it critically for important intellectual content; he has given final approval of the version to be published; and he agrees to be accountable for all aspects of the work in ensuring that questions related to the accuracy or integrity of any part of the work are appropriately investigated and resolved.

Dr Romanenko has made substantial contributions to conception and design; he has been involved in drafting the manuscript or revising it critically for important intellectual content; he has given final approval of the version to be published; and he agrees to be accountable for all aspects of the work in ensuring that questions related to the accuracy or integrity of any part of the work are appropriately investigated and resolved.

Dr Allegri has made substantial contributions to the conception and design, and analysis and interpretation of data; he has been involved in drafting the manuscript or revising it critically for important intellectual content; he has given final approval of the version to be published; and he agrees to be accountable for all aspects of the work in ensuring that questions related to the accuracy or integrity of any part of the work are appropriately investigated and resolved.

\section{Disclosure}

Dr Roberto Casale reports no conflicts of interest in this work. In the last 3 years, he has received funds from the following companies: Grunenthal; Merck; Mundipharma; and GB-Pharma. Dr Massimo Allegri reports no conflicts of interest in this work. In the last 3 years, he has received funds from the following companies: Covidien; Grunenthal; Merck; Mundipharma; and Prostrakan. Dr Yuriy Romanenko reports no conflicts of interest in this work.

\section{References}

1. Treede RD, Jensen TS, Campbell JN, et al. Neuropathic pain: redefinition and a grading system for clinical and research purposes. Neurology. 2008;70(18):1630-1635.
2. Mick G, Baron R, Finnerup NB, et al. What is localized neuropathic pain? A first proposal to characterize and define a widely used term. Pain Manag. 2012;2(1):71-77.

3. Attal N, Cruccu G, Baron R, et al; European Federation of Neurological Societies. EFNS guidelines on the pharmacological treatment of neuropathic pain: 2010 revision. Eur J Neurol. 2010;17(9):1113-e88.

4. Dworkin RH, O'Connor AB, Backonja M, et al. Pharmacologic management of neuropathic pain: evidence-based recommendations. Pain. 2007;132(3):237-251.

5. Comer AM, Lamb HM. Lidocaine patch 5\%. Drugs. 2000;59(2): 245-249; discussion 250-251.

6. Galer BS, Rowbotham MC, Perander J, Friedman E. Topical lidocaine patch relieves postherpetic neuralgia more effectively than a vehicle topical patch: results of an enriched enrollment study. Pain. 1999;80(3): 533-538.

7. Mick G, Correa-Illanes G. Topical pain management with the $5 \%$ lidocaine medicated plaster - a review. Curr Med Res Opin. 2012;28(6): 937-951.

8. Campbell RL, Parks KW, Dodds RN. Chronic facial pain associated with endodontic therapy. Oral Surg Oral Med Oral Pathol. 1990;69(3): 287-290.

9. Okeson JP. Orofacial Pain: Guidelines for Assessment, Diagnosis and Management. 3rd ed. Chicago, IL: Quintessence; 2006.

10. de Leeuw R. Orofacial Pain: Guidelines for Assessment, Diagnosis, and Management. Hanover Park, IL: Quintessence; 2008

11. Rehm S, Binder A, Baron R. Post-herpetic neuralgia: 5\% lidocaine medicated plaster, pregabalin, or a combination of both? A randomized, open, clinical effectiveness study. Curr Med Res Opin. 2010;26(7): 1607-1619.

12. Lam VY, Wallace M, Schulteis G. Effects of lidocaine patch on intradermal capsaicin-induced pain: a double-blind, controlled trial. J Pain. 2011;12(3):323-330.

13. Casale R, Mattia C. Building a diagnostic algorithm on localized neuropathic pain (LNP) and targeted topical treatment: focus on 5\% lidocainemedicated plaster. Ther Clin Risk Manag. 2014;10:259-268.

14. Rowbotham MC, Davies PS, Verkempinck C, Galer BS. Lidocaine patch: double-blind controlled study of a new treatment method for post-herpetic neuralgia. Pain. 1996;65(1):39-44.

15. Casale R, Di Matteo M, Minella CE, Fanelli G, Allegri M. Reduction of painful area as new possible therapeutic target in post-herpetic neuropathic pain treated with 5\% lidocaine medicated plaster: a case series. J Pain Res. 2014;7:353-357.

16. Sessle BJ, Hu JW, Amano N, Zhong G. Convergence of cutaneous, tooth pulp, visceral, neck and muscle afferents onto nociceptive and non-nociceptive neurones in trigeminal subnucleus caudalis (medullary dorsal horn) and its implications for referred pain. Pain. 1986;27(2): 219-235.

17. Gammaitoni AR, Alvarez NA, Galer BS. Pharmacokinetics and safety of continuously applied lidocaine patches 5\%. Am J Health Syst Pharm. 2002;59(22):2215-2220.

18. Baron R, Hans G, Dickenson AH. Peripheral input and its importance for central sensitization. Ann Neurol. 2013;74(5):630-636.

19. Chong MS, Smith TE, Hanna M. Case reports - reversal of sensory deficit associated with pain relief after treatment with gabapentin. Pain. 2002;96(3):329-333.

20. Correa-Illanes G, Calderón W, Roa R, Piñeros JL, Dote J, Medina D. Treatment of localized post-traumatic neuropathic pain in scars with 5\% lidocaine medicated plaster. Local Reg Anesth. 2010;3:77-83. 
Journal of Pain Research

\section{Publish your work in this journal}

The Journal of Pain Research is an international, peer-reviewed, open access, online journal that welcomes laboratory and clinical findings in the fields of pain research and the prevention and managemen of pain. Original research, reviews, symposium reports, hypothesis formation and commentaries are all considered for publication.
Dovepress

The manuscript management system is completely online and includes a very quick and fair peer-review system, which is all easy to use. Visit http://www.dovepress.com/testimonials.php to read real quotes from published authors.

\footnotetext{
Submit your manuscript here: http://www.dovepress.com/journal-of-pain-research-journal
} 